\title{
Partial Hysterectomy
}

National Cancer Institute

\section{Source}

National Cancer Institute. Partial Hysterectomy. NCI Thesaurus. Code C40961.

Surgical removal of part of the uterus. 